\title{
The Study of Plate Number Recognition for Parking Security System
}

\author{
Shakeeb M.A.N. Abdul Samada, Fahri Heltha*ab, M. Faliqa \\ aUCSI University, Kuala Lumpur, Malaysia \\ ${ }^{b}$ Unsyiah, Banda Aceh,Indonesia
}

\begin{abstract}
Car Plate Number Recognition System is an important platform that can be used to identify a car vehicle identity. The Recognition System is based on image processing techniques and computer vision. A common webcam is used to capture an image of the car plate number from different distance, and the identification process is conducted through four stages: Image Acquisition \& Preprocessing, Extraction, Segmentation, and Character Recognition. The Acquisition \& Pre-processing stage is extracted the region of interest of the image. The image is captured by live video of the webcam, then converted to grayscale and binary image. The Extraction stage is extracted the plate number characters from binary image using a connected components method. The Segmentation stage is done by implementing horizontal projection as well as moving average filter. Lastly, in the Character Recognition, is identified the segmented characters of the plate number using optical character recognition. The proposed method is worked well for Malaysian's private cars plate number, and can be implemented and installed at any automatic car park systems to increase level of security by confirming between the bar code of the parking ticket and the plate number of the car at the incoming and outgoing gates.
\end{abstract}

Keywords: image recognition system; acquisition \& pre-processing; extraction; segmentation; character recognition

\section{Introduction}

The number of vehicles is increasing day by day, and it is getting more and more complicated to manage vehicles. The frequently asked question is how to recognize a particular vehicle? A clear answer to this question is by using car plate number identification. Similarly, this project is identifying all vehicles that entering and exiting the parking lots using their plate number, the system increases the level of the security in the parking lots by assigning parking ticket based on the plate number of the vehicle. Car Plate Number Recognition system is performed to automatically detect and recognize car plate number to help human. Previously, the car plate number was watched manually by a human. So, this project is developing to substitute human to observe the car and automatically capture the image. Besides that, the system is developed to detect and recognize Car Plate Number, also it is developed to match between Car Plate Number and barcode of the ticket in the parking lots to increase the security of parking lots.

Car Plate Number Recognition System is a process which the vehicles are recognized and identified using their plate numbers. The system is using image processing techniques that extracted the plate and recognized the characters of the car plate from the image [1]. The main functions of the hardware components are car plate detection, car plate image acquisition, and data transmission. The software components take care of vehicle image pre-processing, car plate localization, character 
segmentation, and in the end character recognition [2]. It works by extracting and segmenting the characters of the car plate number from an image. Many research had already been done for the recognition of Korean, European, Chinese, American and other car plates. From a specific point of view, Car Plate Number Recognition system is an important technique which is used in Intelligent Transportation System. Car Plate Number Recognition system is an advanced technology that no need direct human intervention to identify the vehicles by their number plates [3].

There are two varieties of Car Plate Number Recognition system: Online Car Plate Number Recognition system and Offline Car Plate Number Recognition system. In the Online Car Plate Number Recognition system, the extraction and recognition of car plates take place instantaneously from a live video, enabling real time tracking of vehicles through the surveillance camera. On the other hand, the Offline Car Plate Number Recognition system processing an image of the vehicle that already captured and stored to the database file for further processing and interpretation of vehicle car plates [4]. The software that used to impermeant the system is MATLAB which provides image processing tools.The software allows developer to work interactively with different data, helps the developer to keep track of files and variables, and simplifies common programming.

This study only focused on the Malaysian private car plate number and implemented them to automatic car park system to increase the level of security of the parking system.

\section{Research Methodology}

The proposed approach for Smart Car Plate Number Recognition System is represented in Figure 1. It is divided into four stages which are image acquisition \& pre-processing, car plate number extraction, car plate number segmentation, and character recognition.

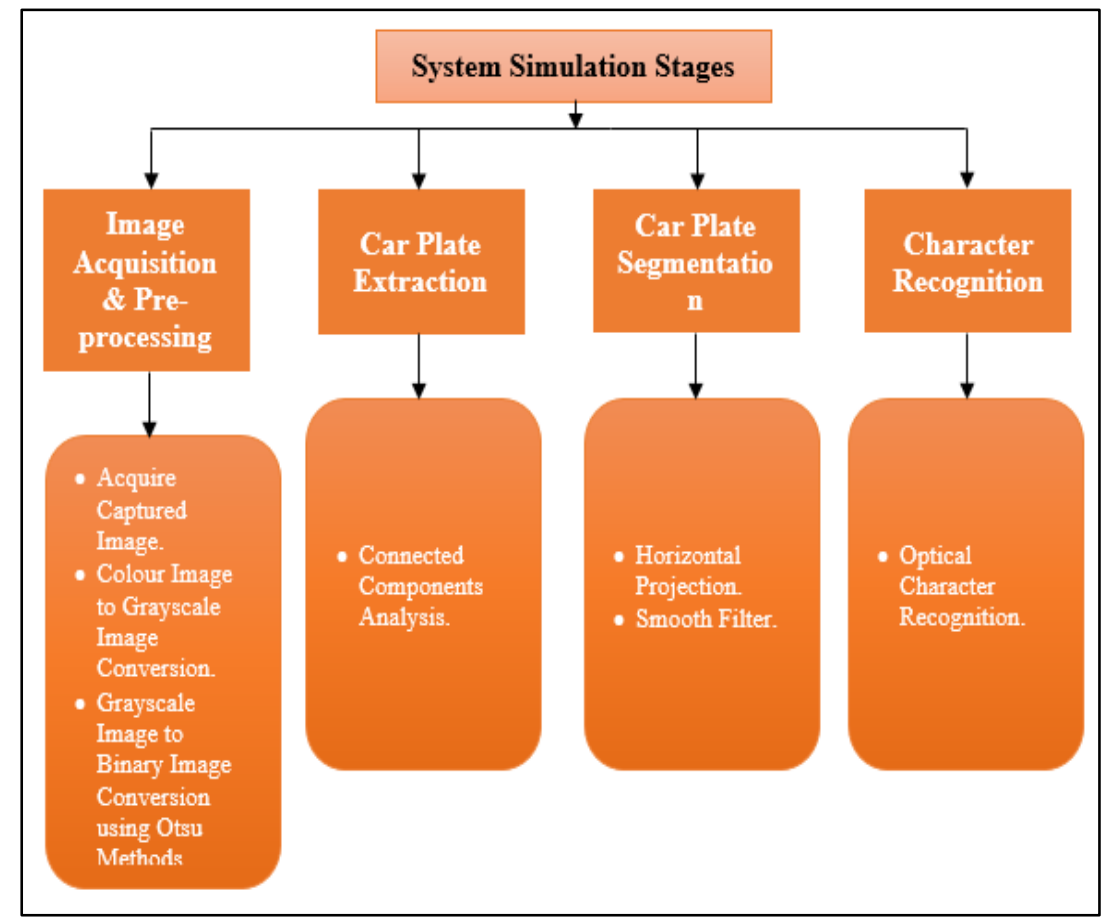

Figure 1. Proposed approach for system simulation stages

\subsection{Image acquisition \& pre-processing}

The image acquisition is conducting in three steps. The first step is connecting the Webcam that will be used to capture an image of the car plate number. The captured image is a coloured image and needs to be converted into Grayscale image. The image contains 3 layers, which are each pixel consist of 3 colours of Red, Green, and Blue with the colour value for each pixel are in $0-255$, respectively. The Conversion process from grayscale to binary is reducing the value of the grayscale from $0-255$ to $0-1$ to be in only two possible values for each pixel i.e. 1 for White or 0 for Black. Then, thresholding, one of the methods that can be used for image segmentation and conversion to binary image, is giving each pixel in the grayscale image to be marked as an object. If it is larger than the other threshold, 
the object will be assumed to be stronger than the background pixels. The object will be assigned as white pixel and the background will be assigned as black pixel in the binary image. Next process, Histogram, is generating total number of pixels from grayscale image. The Otsu method formulas are implementing to find the different between the foreground variance and the background variance of the grayscale image.

\subsection{Plate extraction}

Malaysian's private cars have a plate number that is black background and white characters. In this stage, an algorithm called Connected Components Analysis is used to extract the characters of the car plate from the binary image. The reason for extracting the characters rather than the car plate is that after converting the grayscale image to binary image the characters will appear as an object and the background of the car plate number will appear as a black pixel. Moreover, the Connected Components Analysis method is used to extract the white objects from the binary image. This method will be used to extract the characters from the binary image. The following table shows the total number of connected white pixels for each character at the nearest and the furthest point to the camera of this project.

Table 1. Connected white pixels for each character

\begin{tabular}{|c|c|c|c|}
\hline $\begin{array}{l}\text { Car Plate } \\
\text { Number }\end{array}$ & Characters & $\begin{array}{c}\text { Number of Connected } \\
\text { White Pixels at The } \\
\text { Nearest Position }\end{array}$ & $\begin{array}{l}\text { Number of Connected } \\
\text { White Pixels at The } \\
\text { Furthest Position } \\
\end{array}$ \\
\hline \multirow{6}{*}{ SDM 379} & & 9391 & 2538 \\
\hline & & 10086 & 2807 \\
\hline & & 14192 & 3994 \\
\hline & 3 & 8520 & 2382 \\
\hline & & 6553 & 1816 \\
\hline & & 10001 & 2739 \\
\hline \multirow{6}{*}{ WYH 4366} & $i$ & 12679 & 3653 \\
\hline & N & 6797 & 1960 \\
\hline & & 9110 & 2732 \\
\hline & 4 & 8165 & 2455 \\
\hline & 3 & 8000 & 2329 \\
\hline & 6 & 9384 & 2707 \\
\hline \multirow{6}{*}{$\begin{array}{l}\text { Jak } \\
9936\end{array}$} & & 7146 & 1809 \\
\hline & (1) & 12987 & 3261 \\
\hline & 1 & 11957 & 2979 \\
\hline & 9 & 10195 & 2689 \\
\hline & 3 & 8820 & 2315 \\
\hline & (๑) & 10410 & 2710 \\
\hline
\end{tabular}

\subsection{Plate segmentation/ character segmentation}

The binary image still contains some undesirable objects, after extracting process. It still consists of noise, unwanted objects, next to the characters. The objects have the same number of white pixels as the extracted characters, which means the numbers are between 14192 and 1816 and some noises 
are still between that range. The characters will be segmented and the undesirable objects will be removed as much as possible. An algorithm called Projection is used to detect/segment the car plate number and its characters. Furthermore, the algorithm can be used horizontally and vertically to segment the car plate number. Horizontal and Vertical Projection is a method that scans the binary image and finds the sum of the white pixels in Y-axis (Horizontal) or X-axis (Vertical) and plot it to a graph. In this project, Horizontal Projection will be used to detect and segment the characters of the plate number.

\subsection{Character recognition}

Character Recognition is the last stage of Smart Car Plate Number Recognition System, where the segmented image will be used for character recognition. A method called Optical Character Recognition will be used in this step to recognize the characters. This algorithm uses the same techniques as Template Matching. The Template Matching means use the image of the characters in the database to match it with the segmented characters from the binary image, and the segmented characters must be in the same resolution as the characters in the database. Optical Character Recognition (OCR) has advantages over the Template Matching, where it does not need the characters to be segmented from the binary image and the resolution of the segmented characters will not affect the character recognition. Furthermore, the OCR can recognize the Punctuation from the image.

\section{Results and Discussions}

\subsection{Hardware}

The hardware prototype shown in Figure 2 is made of wood and coated with black tape to match the background of Malaysia's car plate number. The measurements of the hardware were assumed for several reasons: to place the camera of the system in a position that is higher than the car plate number to prevent light reflection which may cause character recognition failure, to let the car plate number stands in a straight position, to allow the car plate number changing its position to be more realistic to the parking gate system. A slider had been made to allow the camera of Smart Car Plate Number Recognition System capturing an image of the car plate number from various distance.

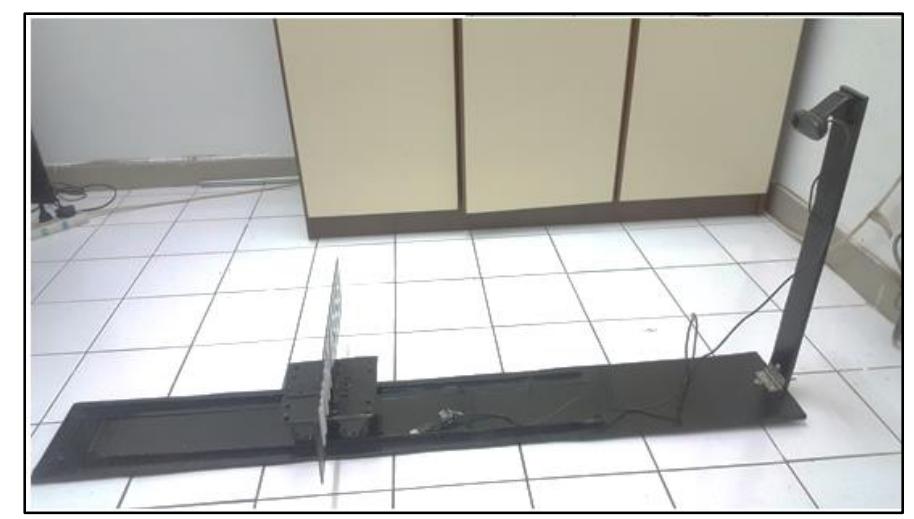

Figure 2. System hardware

\subsection{Software and algorithm}

\section{a. Image Acquisition \& Pre-processing}

The captured image contains 3 layers with the colour value for each pixel are 0-255 Red, 0-255 Green, $0-255$ Blue. The Conversion from grayscale to binary is conducted to reduce the value of the grayscale from $0-255$ to $0-1$ based on the histogram of the grayscale image and the binary image which result only two possible values for each pixel 1 (White) or 0 (Black). 


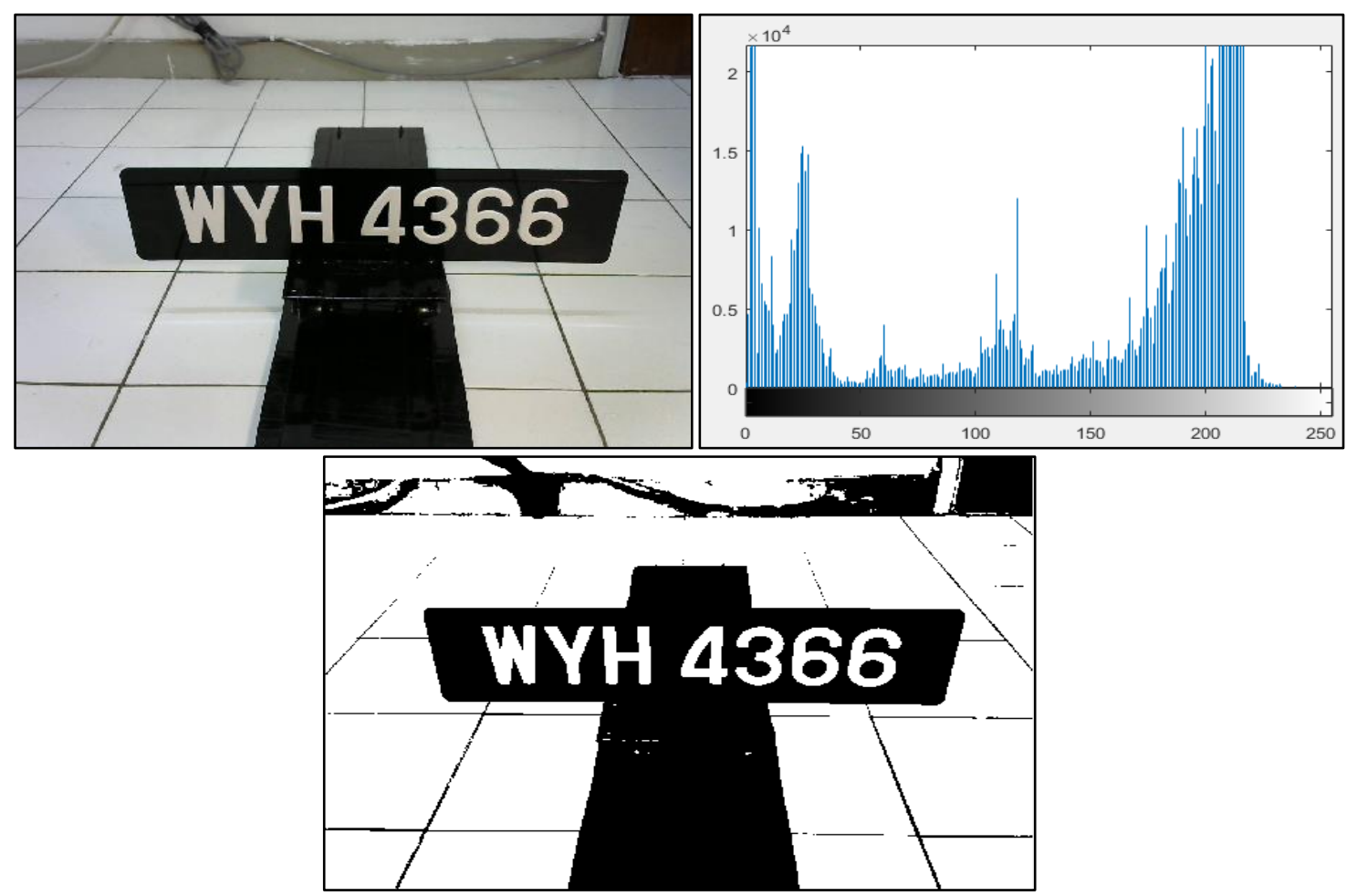

Figure 3. Colour to grayscale to binary

\section{b. Plate extraction}

Connected Components Analysis method is used to extract the characters of the car plate number from the binary image. It will remove the connected white pixels that have fewer than 16000 pixels from the binary image. The number 14192 was selected from the table in the previous chapter. The table shows that 14192 is the maximum connected white pixels in the nearest position, and the assumption of the filter is based on the number as shown in Figure 4, the binary image after applying the filter_1.

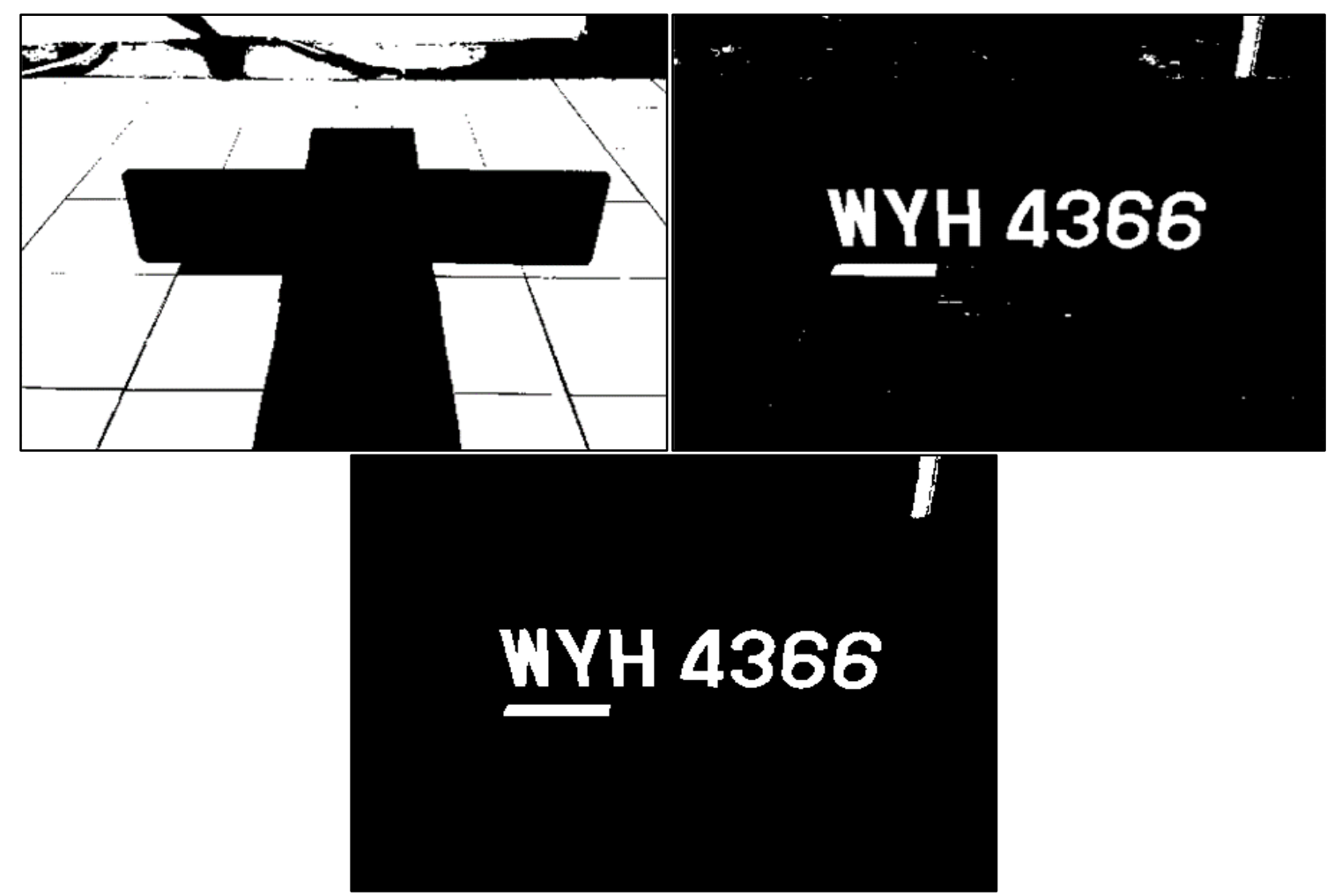

Figure 4. Plate extraction 


\section{c. Plate and character segmentation}

The characters of the plate number are segmented from the binary image using Horizontal Projection method. It scans the binary image horizontally and counts the number of white pixels in each row. The methods, herewith, are segmented the characters of the plate number and smooth the represented data of the horizontal projection. The characters of the plate number is segmented from the binary image by taking the maximum value from the graph and multiply it by 0.5 . In this paper, the maximum value is 348.13 and the average is 174.06 .

\section{d. Character recognition}

In this stage, the segmented characters can be recognized by OCR function in MATLAB. OCR is a tool in MATLAB that have the ability to recognize characters from an image. Furthermore, the database of the OCR contains a trained data of characters and numbers. In this project, OCR function is used in this project to recognize characters and numbers only. OCR takes the objects individually from the binary image, where every object is compared with objects in database of the OCR. After comparing the object with all objects in the database, and the maximum match is selected. Character confident is used in this stage to ignore the objects that matches the database with less than $60 \%$, where the objects that matches the character or the number with more than $60 \%$ will be selected.

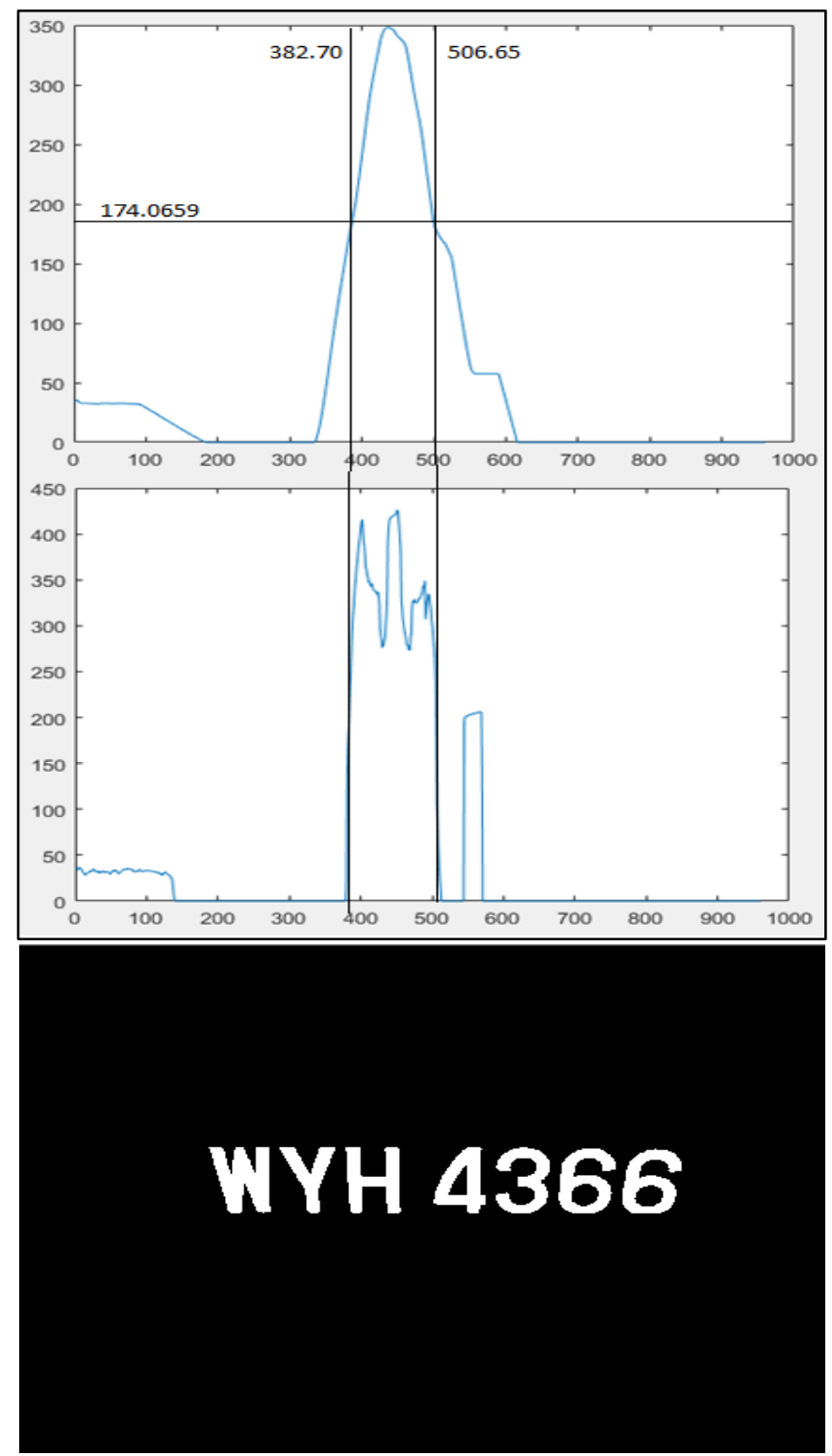

Figure 5. Plate segmentation 


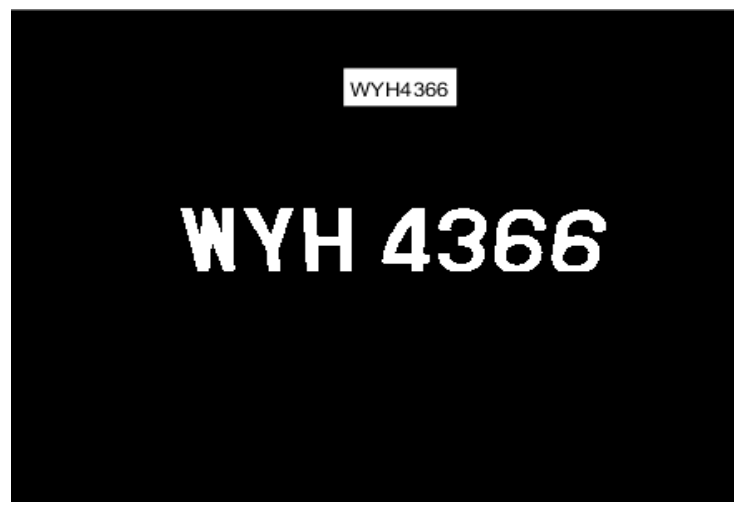

Figure 6. Character recognition

\subsection{Graphical User interface (GUI)}

The GUI shown in Figure 7, is designed as a friendly user interface to recognize the plate number and apply the ticket barcode for each car who are entering the parking lots. The things will be visible in the GUI. The webcam captured images of the plate numbers are also shown in the GUI. Four (4) pushbuttons are created in GUI as a start previewing the live video from the webcam, a stop previewing the live video from the webcam, a command to capture image of the car plate number from the live video, as a command to capture an image of the car plate number from the live video and recognizing the characters, as a function after entering the barcode to confirm that it pairs for the plate number who entering the gate. The characters of the car plate number and the barcode will be compared with the database of the cars that entered the parking lots.

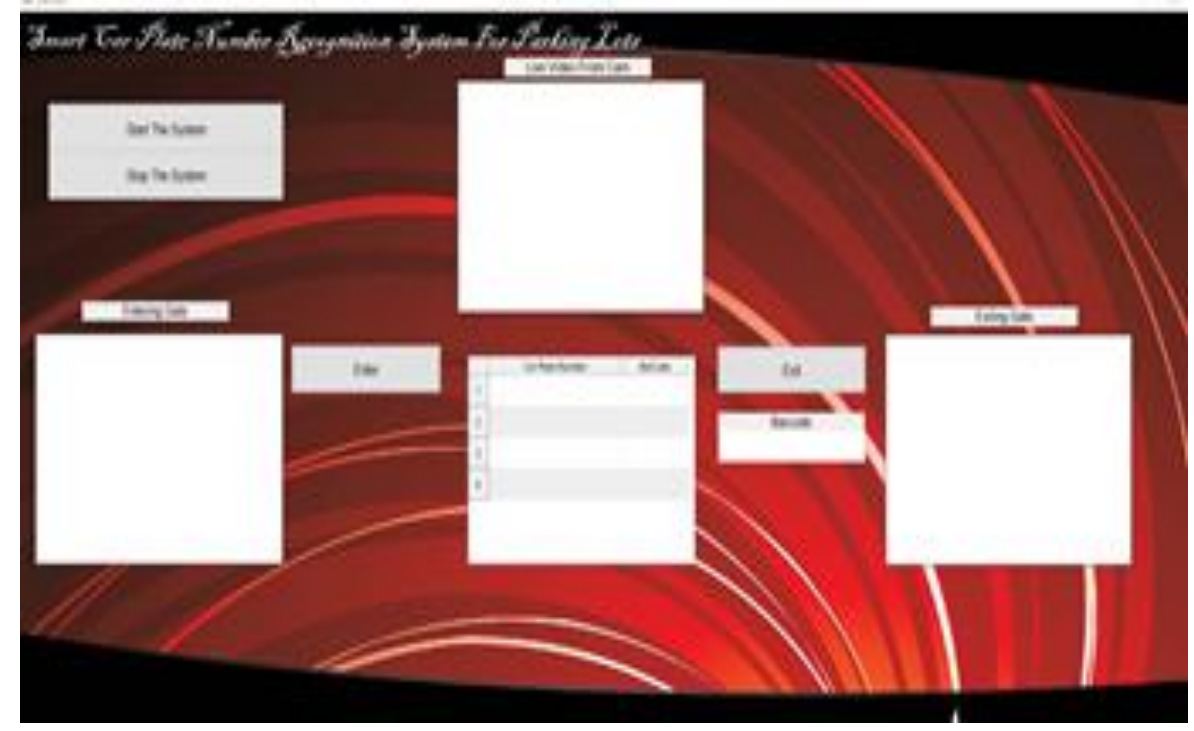

Figure 7. System GUI

Table 2. System efficiency

\begin{tabular}{ccccc}
\hline Car Plate Number & Efficiency & Tested Times & Correct & Error \\
\hline SDM 379 & $90 \%$ & 20 & 18 & 2 \\
WYH 4366 & $95 \%$ & 20 & 19 & 1 \\
JQK & & & & \\
9936 & $80 \%$ & 20 & 16 & 4 \\
\hline
\end{tabular}

\subsection{Performance accuracy of the system}

The accuracy results of comparison between the plate number images and bar codes have been tested. An image of the car plate number was captured multiple times from different angles and distances. Table 2 shows the accuracy and the efficiency of the system. 


\section{Conclusions}

The paper discussed the principle of a Smart Car Plate Number Recognition System using image processing techniques and its application to increase level of security of the existing automatics car park systems. The proposed system has recognized well for the car plate number with white colour characters and black background, such as Malaysian's private cars plate number system. In addition, it has been designed to recognize the plate number from flexible distance, from 50 to $122 \mathrm{~cm}$, by common webcam cameras. The system has been combined with bar codes reading system used in the gate of the parking lots, to be compared and paired with each plate numbers entering the parking lots and to be verified them in the exiting gate. The recognition techniques of the image has been implemented succesfully to the car plate number identifications. The algorithm for Smart Car Plate Number Recognition System has been developed succesfully for Malaysian's private plate number, which has included four stages process in modules: Image Acquisition \& Pre-processing, Car Plate Number Extraction, Character Segmentation, and Character Recognition. The performance analysis has shown a very accurate results in capturing the image of the plate number in various distances and in different light conditions, it has recognized the characters of the plate number clearly up to 95\% accuracy. The matching process between the plate number and barcode, in the incoming and exitting gates of automatic cara park systems, has been succesfully identified and confirmed. It will surely gaining the level of the security of the parking systems. The accuracy rate of the matching process experiments between the plate number and the related barcode have shown the average results of identification and confirmation up to $88.33 \%$.

\section{References}

[1] Abechi, S.E. Gimba, C.E. Uzairu A. and Dallatu, Y.A. Preparation and Characterization of Activated Carbon from Palm Kernel Shell by Chemical Activation. Research Journal of Chemical Sciences, 2013;3(7):54-61.

[2] Amir Ebrahimi, A. A. (2015). Car License Plate Recognition using Color Features of Persian License Plates. Journal of Advances in Computer Research.

[3] Jin, L. X. (2012). License plate recognition algorithm for passenger cars in Chinese residential areas. . Jin, L., Xian, H., Bie, J., Sun, Y., Hou, H., \& Niu, Q. (2012). License plate recognition algorithm for passenger cars in Chinese residential areas. Sensors, 8355-8370.

[4] Prashant Chaudhary, D. V. (2016). Automatic License Plate Recoganization System Using LabVIEW: Review. International Journal of Advanced Research in Computer Science and Software Engineering.

[5] Suprokash Dey, A. C. (2014). An Efficient Technique to Recognize License Plate using Morphological Edge Detection and Character Matching Algorithm. International Journal of Computer Applications, 0975 - 8887.

[6] Mr. G. T. Sutar, P. M. (2014). Number Plate Recognition Using an Improved Segmentation. International Journal of Innovative Research in Science, Engineering and Technology, 2319-8753.

[7] Kaur, S. (2016). An Automatic Number Plate Recognition System under Image Processing. I.J. Intelligent Systems and Applications, 14-25.

[8] S.Kranthi, K. A. (2011). Automatic Number Plate Recognition. International Journal of Advancements in Technology, 0976-4860.

[9] Amr Badr, M. M. (2011). Automatic Number Plate Recognition System. Annals of the University of Craiova, Mathematics and Computer Science Series, 62-71.

[10] Varalakshmi, D. L. (2015). Recognition of License Plate Numbers Using Image Processing Technique and Genetic Algorithm. International Journal of Engineering Research and General Science.

[11] Kaur, S. (2016). An Automatic Number Plate Recognition System under Image Processing. I.J. Intelligent Systems and Applications, 14-25.

[12] Sharma, R. (2015). Automatic License Plate Based Smart Vehicle Validation \&Security by Gate Control \&Email Send. International Journal of Computer Science and Information Technologies. 